\title{
Robustness of Reduced Order Non-Parametric Model for Inverse ECG Solution Against Modeling and Measurement Noise
}

\author{
Onder Nazim Onak ${ }^{1}$, Yesim Serinagaoglu Dogrusoz ${ }^{1}$, Gerhard Wilhelm Weber ${ }^{1,2}$ \\ ${ }^{1}$ Middle East Technical University, Ankara, Turkey \\ ${ }^{2}$ Poznan University of Technology, Poznan, Poland
}

\begin{abstract}
Spline-based methods have been applied to inverse problems in science and engineering. Those studies have shown that if proper spline bases can be chosen, problem complexity can be significantly reduced while increasing estimation accuracy and robustness against the disturbances. We proposed non-parametric regression spline based approach for the solution of inverse ECG problem and assessed its robustness against measurement noise, variation of the heart size from its true size, and their combination. Our model defines the spline functions in terms of spatial coordinate variables based on the given epicardial surface geometry. The results demonstrated that, proposed method performed better than the Tikhonov regularization and can be feasible alternative for the inverse ECG problem.
\end{abstract}

\section{Introduction}

Inverse problem of ECG can be described as inferring cardiac electrical activity from the given body surface potential measurements (BSPM) and mathematical model that characterizes the relationship between measurements and sources. Here, the mathematical model is constructed by solving the forward ECG problem which mainly dependent on size, location and electrical properties of the heart, torso and other internal structures between the heart and torso surfaces. However, due to dispersing effect of the torso and the discretization process, this inverse problem has an ill-posed nature. The term ill-posed inverse problem often stands for problems that have either no solution or many solutions in the desired class, or alternatively that the solution is unstable. But in common use, the term ill-posed is related to unstable problems [1]. From the perspective of inverse ECG problem, instability means that relatively small changes in the body surface measurements are abundantly amplified in the solution. Consequently, problem needs to be appropriately constrained by introducing prior information about the solution in order to obtain physio- logically meaningful outcomes.

Spline-based methods have been applied to ill-posed inverse problems in various fields of science and engineering including electrocardiography $[2,3]$. The main advantages of the spline-based methods are the parametrization of the problem in terms of a small number of unknowns and changing the approximation in local regions without affecting remote portions of the curve or the surface that we wish to approximate. These properties improve the robustness of the inversion and increase the accuracy of the reconstruction [4]. In this context, the constructed model for the unknown parameter of the inverse problem is the projection of data onto the space spanned by the selected spline basis in the model. The aforementioned applications of splines in inverse problems use parametric methods. In other words, assumptions on functional relationship between dependent and independent variables must be specified in advance. Typical approach to choose these parameters is quite arbitrary by using trial-and-error [5].

The method that we considered in this study is the continuation of our previous work [6] and it is simplified by reducing number of constraints. Proposed approach solves the inverse ECG problem by using non-parametric regression technique. Different than the parametric approaches, non-parametric spline-based method allowed us to select proper splines for the representation of unknown epicardial potential distribution step by step in an algorithmic way by using available measurements and supplied epicardial surface geometry. As a non-parametric method we modified and utilized Multivariate Adaptive Regression Splines (MARS) [7] for the solution of the inverse electrocardiography problem considering the temporal and spatial evolution of the epicardial potentials. Our model defines the spline functions in terms of spatial parameters based on the given epicardial surface geometry, but the selection of those splines for the model is dependent on the defined lack-of-fit criteria. Thus, any change in geometry or measurements can alter the constructed model for the purpose of obtaining an accurate estimate. Consequently, the constructed epicardial potential distribution model may pos- 
sess different functional relationships at each time instant in order to obtain a model that yields minimum lack-of-fit error.

In this study, we carried out simulations with a combination of measurement noise and geometry error (the size of the heart) and evaluated their effects on the proposed MARS-based method. Estimation results were compared with the zero-order Tikhonov regularization solutions.

\subsection{MARS}

Multivariate Adaptive Regression Splines (MARS) proposed by Friedman [7], is a non-parametric regression procedure. Different than the parametric approaches, nonparametric methods do not make any specific assumption about the structure of the regression function and tries to estimate the form of the relationship between dependent and independent variables [8].

MARS is an adaptive procedure because the selection of the basis functions (BFs) is data-based and specific to the given problem at hand. A special advantage of MARS lies in its ability to estimate the contributions of the basis functions so that both the additive and the interactive effects of the predictors are allowed to determine the response variable. MARS uses expansions in piecewise linear one-dimensional basis functions of the form $(v-\tau)_{+}$ and $(\tau-v)_{+}$, where " $(\cdot)_{+}$"means the positive part:

$$
\begin{aligned}
& (v-\tau)_{+}= \begin{cases}v-\tau, & \text { if } v>\tau, \\
0, & \text { otherwise, }\end{cases} \\
& (\tau-v)_{+}= \begin{cases}\tau-v, & \text { if } v<\tau, \\
0, & \text { otherwise. }\end{cases}
\end{aligned}
$$

The relation between the input and the response in the general model is expressed as:

$$
T=f(\mathbf{V})+\varepsilon,
$$

where $T$ is a response variable, $\mathbf{V}=\left(\mathbf{V}_{\mathbf{1}}, \mathbf{V}_{\mathbf{2}}, \ldots, \mathbf{V}_{\mathbf{p}}\right)^{\mathbf{T}}$ is a vector of predictors and $\varepsilon$ is the additive stochastic error term in the observation with zero mean and finite variance. MARS builds reflected pairs for each input $V_{j}(j=1,2, \ldots, p)$ with $p$-dimensional knots $\tau_{\mathbf{i}}=$ $\left(\tau_{\mathbf{i} 1}, \tau_{\mathbf{i} 2}, \ldots, \tau_{\mathbf{i p}}\right)^{\mathbf{T}}$ at, or just nearby, each input data vectors $\tilde{\mathbf{v}}_{\mathbf{i}}=\left(\tilde{\mathbf{v}}_{\mathbf{i} 1}, \tilde{\mathbf{v}}_{\mathbf{i} 2}, \ldots, \tilde{\mathbf{v}}_{\mathbf{i p}}\right)^{\mathbf{T}}$ of that input $(i=$ $1,2, \ldots, N)$. Then, the collection of BFs is:

$$
\begin{array}{r}
\varphi:=\left\{\left(V_{j}-\tau\right)_{+},\left(\tau-V_{j}\right)_{+} \mid \tau \in\left\{\tilde{v}_{1 j}, \tilde{v}_{2 j}, \ldots, \tilde{v}_{N j}\right\},\right. \\
j \in\{1,2, \ldots, p\}\} .
\end{array}
$$

The fundamental idea of MARS is to use products and, then, the combination of the linear truncated basis functions to approximate the model. Thus, the functions of
MARS consist of single spline functions or the product of two or more of the truncated power functions to allow for the interactions.

\section{Reformulation of the Problem}

Proposed method in this study model the potential distribution on the epicardial surface, based on MARS using the heart geometry and the body surface potential values.

We treat and model the potential distribution on the epicardial surface as a function $f(\mathbf{p})$ defined over a 3dimensional epicardial surface. Consequently, the epicardial potential vector $\mathbf{x}=\left[\mathbf{x}_{1}, \mathbf{x}_{2}, \ldots, \mathbf{x}_{\mathbf{N}}\right]^{\mathbf{T}}$ can be expressed as a collection of function values $f(\mathbf{p})$ at predefined coordinates $\mathbf{p}_{\mathbf{i}}(i=1,2, \ldots, N)$ :

$$
x_{i}=f\left(\mathbf{p}_{\mathbf{i}}\right) \quad\left(\mathbf{p}_{\mathbf{i}} \in \Omega\right) .
$$

Here, $\Omega \in \mathbb{R}^{3}$ denotes the 3 -dimensional epicardial surface and $\mathbf{p}$ stands for coordinate vector of any point on this surface. We then write the linear inverse ECG problem as follows:

$$
\mathbf{y}=\mathbf{A x}+\mathbf{n} .
$$

If we treat $y_{i}(i=1,2, \ldots, M)$ as the responses, and $\mathbf{p}_{\mathbf{j}}(j=1,2, \ldots, N)$ as the predictor values, then MARS method can be applied to estimate the function $f(\mathbf{p})$. Thus, the MARS estimate of the unknown function $\hat{f}(\mathbf{p})$ can be written in the following form:

$$
\hat{f}(\mathbf{p})=\theta_{\mathbf{0}}+\sum_{\mathbf{l}=\mathbf{1}}^{\mathbf{L}} \theta_{\mathbf{l}} \psi_{\mathbf{l}}(\mathbf{p}) .
$$

Here, $L$ is the number of basis functions in the model, $\psi_{l}(l=1,2, \ldots, L)$ are BFs from $\varphi$ or products of two or more such functions, $\psi_{l}$ is taken from a set of $L$ linearly independent basis elements, and $\theta_{l}$ are the unknown coefficients for the $l^{\text {th }}$ basis function $(l=1,2, \ldots, L)$ or for the constant $1(l=0)$. In light of the equations given above, the $i^{\text {th }}$ torso measurement $y_{i}$ can be written as:

$$
y_{i}=\sum_{j=1}^{N} a_{i j} \hat{f}\left(\mathbf{p}_{\mathbf{j}}\right)+\mathbf{n}_{\mathbf{i}}
$$

If we substitute Eqn. (7) into Eqn. (8):

$$
y_{i}=\sum_{j=1}^{N} a_{i j}\left(\theta_{0}+\sum_{l=1}^{L} \theta_{l} \psi_{l}\left(\mathbf{p}_{\mathbf{j}}\right)\right)+n_{i} .
$$

Then the Eqn. (6) can be expressed based on spline functions and corresponding coefficients:

$$
\mathbf{y}=\mathbf{A} \boldsymbol{\Psi} \boldsymbol{\theta}+\mathbf{n}
$$

Here, $\boldsymbol{\Psi}$ is a matrix composed by spline bases, which are constructed based on epicardial surface geometry, $\theta$ 
represents a corresponding coefficient vector, $\mathbf{A}$ is the forward transfer matrix, $\mathbf{y}$ is the torso measurements and $\mathbf{n}$ is measurement noise. In other words, the $\boldsymbol{\Psi} \boldsymbol{\theta}$ term is the approximation of the unknown epicardial potential vector.

MARS is a regression procedure to explore the relationships between dependent and independent variables. It assumes there exist no transformation between the measurements and the function we wish to approximate. Thus, MARS algorithm needs to be modified to solve the illposed inverse problem defined in Eqn. (10). Consequently, we replaced the standard MARS algorithm lack-of-fit criteria with the following optimization problem:

$$
\underset{\mathbf{\Psi} \boldsymbol{\theta}}{\operatorname{minimize}}\|\mathbf{y}-\mathbf{A} \boldsymbol{\Psi} \boldsymbol{\theta}\|_{\mathbf{2}}^{\mathbf{2}}+\lambda\left\|\boldsymbol{\Psi} \boldsymbol{\theta}-\hat{\mathbf{x}}_{\mathbf{k}-\mathbf{1}}\right\|_{\mathbf{2}}^{\mathbf{2}} .
$$

Here, $\lambda$ and $\hat{\mathbf{x}}_{\mathbf{k}-\mathbf{1}}$ are the regularization parameter and estimated epicardial potential vector for the previous time instant, respectively. The initial state $\hat{\mathbf{x}}_{\mathbf{0}}$ was taken as the Tikhonov solution at time instant $k=1$.

\subsection{Results and Conclusion}

The effects of modeling inaccuracies and measurement noise are important for the success of applied regularization techniques. Modeling errors originate from several parameters or assumptions that are used in the solution of the forward problem. Variations in the conductivities of torso tissues, segmentation errors of medical images, discretization errors, movement of the heart, geometry parameters such as size, location of the heart and electrode positions on the torso. Common sources of measurements errors are faulty calibration or unpredictable variations in the measuring instruments because of the environmental conditions. These disturbances degrade the estimation accuracy of the regularization methods by generating data different from the exact model parameters and potential values. Therefore, it is important to test robustness of regularization methods against the modeling errors and measurement noise.

In this part of our study, we carried out simulations with combination of geometry error and measurement noise to evaluate the performance of proposed method. In our experiments we only considered the variation of the heart size as a modeling error. The noise free body surface potentials were simulated using forward transfer matrix corresponding to exact heart-torso geometry and then 10, 20 and 30 dB SNR Gaussian noise were to added these potential values. We utilized 2 recordings with 2 different stimulation sites on the epicardial surface. The results were compared with classical zero-order Tikhonov solutions.

Estimation performances for the reconstructed epicardial potential distributions in terms of average $\mathrm{CC}$ values were presented at the Tables 1 and 2 for various noise levels and scaled heart sizes. MARS-based method produced
Table 1. Estimation mean correlations for MARS-based method for different heart sizes and measurement noise levels.

\begin{tabular}{c|ccc}
\hline \hline & \multicolumn{3}{|c}{ Measurement Noise (dB SNR) } \\
Scale factor & 10 & 20 & 30 \\
\hline 0.8 & $0.73 \pm 0.19$ & $0.77 \pm 0.15$ & $0.77 \pm 0.11$ \\
1.0 & $0.74 \pm 0.18$ & $0.79 \pm 0.15$ & $0.83 \pm 0.12$ \\
1.2 & $0.72 \pm 0.20$ & $0.76 \pm 0.13$ & $0.77 \pm 0.12$ \\
\hline \hline
\end{tabular}

Table 2. Estimation mean correlations for Tikhonov method for different heart sizes and measurement noise levels.

\begin{tabular}{c|ccc}
\hline \hline & \multicolumn{4}{|c}{ Measurement Noise (dB SNR) } \\
Scale factor & 10 & 20 & 30 \\
\hline 0.8 & $0.59 \pm 0.21$ & $0.65 \pm 0.18$ & $0.67 \pm 0.15$ \\
1.0 & $0.58 \pm 0.23$ & $0.66 \pm 0.20$ & $0.72 \pm 0.19$ \\
1.2 & $0.58 \pm 0.21$ & $0.64 \pm 0.18$ & $0.67 \pm 0.17$ \\
\hline \hline
\end{tabular}

higher mean $\mathrm{CC}$ values compared to Tikhonov regularization in all experiments. When noise level changed from 30 $\mathrm{dB}$ to $10 \mathrm{~dB}$ SNR, proposed method and Tikhonov regularization performances degraded approximately $11 \%$ and $19 \%$ respectively in terms of mean CC values if no geometric error was imposed into the problem. On the other hand, when the modeling error imposed to the model by changing size of the heart, estimation performances of the MARS-based and Tikhonov methods decreased approximately $5 \%$ and $12 \%$. In addition, if size of the heart were scaled by factors 0.8 and 1.2 both methods estimation accuracies degraded about $7 \%$ when measurement noise levels were $30 \mathrm{~dB}$ SNR. However, according the results the effects of measurement noise dominates estimation quality while noise SNR is increasing.

Table 3. Average activation time Pearson correlation coefficients of MARS-based method for different heart sizes and measurement noise levels.

\begin{tabular}{c|ccc}
\hline \hline & \multicolumn{3}{|c}{ Measurement Noise (dB SNR) } \\
Scale factor & 10 & 20 & 30 \\
\hline 0.8 & 0.89 & 0.93 & 0.92 \\
1.0 & 0.90 & 0.95 & 0.93 \\
1.2 & 0.90 & 0.91 & 0.91 \\
\hline \hline
\end{tabular}

Activation times provide information about the propagation pattern of the reconstructed epicardial potentials. This information could be useful for calculating propagation velocity in different parts of the heart surface and determining the stimulation site. In order to evaluate the estimated 
Table 4. Average activation time Pearson correlation coefficients of Tikhonov method for different heart sizes and measurement noise levels.

\begin{tabular}{c|ccc}
\hline \hline & \multicolumn{3}{|c}{ Measurement } \\
Scale factor & 10 & 20 & 30 \\
\hline 0.8 & 0.60 & 0.89 & 0.92 \\
1.0 & 0.82 & 0.88 & 0.93 \\
1.2 & 0.61 & 0.75 & 0.84 \\
\hline \hline
\end{tabular}

activation times, we calculated the average Pearson CC values corresponding to each method given in the Tables 3 and 4 . Estimated activation times were very close to true ones and obtained high CC values, $\geq 0.90$ on the average, for the proposed MARS-based method in all heart scales and measurement noise levels. On the other hand, combination of heart size error and measurement noise caused significant degradation on the Tikhonov regularization outputs.

\subsection{Discussions and Future Works}

In this study we examined the effects of variation in size of the heart from its true size combined with measurement noise on the proposed adaptive spline-based solution technique. Proposed method yielded more accurate estimations and stayed robust against the combination of geometry error and measurement noise compared to Tikhonov regularization. Our study is continuing using larger set of experimental data to comprehensively understand and investigate the effects of various disturbances.

\section{Acknowledgements}

The authors would like to thank Dr. Robert S. MacLeod from University of Utah, Nora Eccles Harrison Cardiovascular Research and Training Institute for the data used in this study. The implementation of proposed method in this work became possible by MARS software library, AresLab, which was developed by Gints Jekabsons, Dr.sc.ing. from Riga Technical University Institute of Applied Computer Systems.

\section{References}

[1] Kabanikhin, S. I. (2008). Definitions and examples of inverse and ill-posed problems. Journal of Inverse and IllPosed Problems, 16(4).

[2] Baussard, A., Miller, E. L., Prémel, D. (2004). Adaptive B -spline scheme for solving an inverse scattering problem. Inverse Problems, 20(2), 347-365.

[3] Erem, B., Coll-Font, J., Orellana, R. M., St'Ovicek, P., Brooks, D. H. (2014). Using transmural regularization and dynamic modeling for noninvasive cardiac potential imaging of endocardial pacing with imprecise thoracic geometry. IEEE Transactions on Medical Imaging, 33(3), 726-738.

[4] Baussard, A., Miller, E. L., Lesselier, D. (2004). Adaptive multiscale reconstruction of buried objects. Inverse Problems, 20(6), S1-S15.

[5] Harmening, C., Neuner, H. (2016). Choosing the optimal number of B-spline control points (Part 1: Methodology and approximation of curves). Journal of Applied Geodesy, 10(3).

[6] Onak, Ö. N., Serinagaoglu Dogrusöz, Y., Weber, G. W. (2017). Effect of the geometric inaccuracy in MARS-based inverse ECG solution approach. In Computing in Cardiology (CinC), 2017. Rennes -France.

[7] Friedman, J. H. (1991). Multivariate adaptive regression splines. The Annals of Statistics, 19(1), 1-67.

[8] Gyorfi, Laszlo, Michael Kohler, Adam Krzyzak, and H. W. (2002). A Distribution-Free Theory of Nonparametric Springer Series in Statistics. New York: Springer-Verlag. ISBN 0387954414.

Address for correspondence:

O. N. Onak

Institute of Applied Mathematics, METU Universiteler Mahallesi Dumlupınar Bulvari No:1 06800 Ankara, Turkey

ononak@gmail.com 